\title{
Long-term outcome after EUS-guided radiofrequency ablation: Prospective results in pancreatic neuroendocrine tumors and pancreatic cystic neoplasms
}

\section{다)(i) $\odot=$}

\author{
Authors \\ Institutions \\ 1 Hopital Nord - Gastroenterology, Marseille, France \\ 2 Paoli Calmettes Institute - Endoscopic Unit, Marseille, \\ France \\ 3 Hôpital St-Joseph - Gastroenterology, Marseille, France \\ 4 Centre Chirurgical Lyon Mermoz - Endoscopy Unit, Lyon, \\ France \\ 5 CHRU Minjoz, Besancon, French Polynesia \\ 6 Hôpital de L'Archet 2 - Pôle digestif, Nice France
}

Marc Barthet ${ }^{1}$, Marc Giovannini' ${ }^{2}$, Mohamed Gasmi ${ }^{1}$, Nathalie Lesavre ${ }^{1}$, Christian Boustière ${ }^{3}$, Bertrand Napoleon ${ }^{4}$, Arthur LaQuiere ${ }^{3}$, Stephane Koch ${ }^{5}$, Geoffroy Vanbiervliet ${ }^{6}$, Jean-Michel Gonzalez ${ }^{1}$

submitted 26.8.2020

accepted after revision 29.10.2020

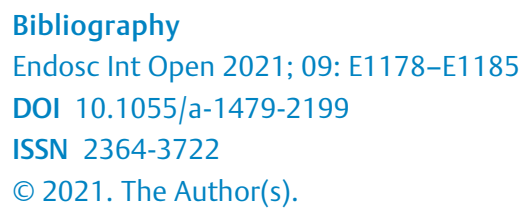

\section{ABSTRACT}

Background and study aims Endoscopic ultrasoundguided radiofrequency ablation (EUS-RFA) for pancreatic neuroendocrine tumors (NETs) and intraductal pancreatic mucinous neoplasia (IPMN) with worrisome features or high-risk stigmata (WF/HRS) has been evaluated in few series with short-term outcomes. This study's primary endpoint was to assess the long-term efficacy of EUS-RFA in patients with NETs or pancreatic cystic neoplasms (PCNs) over at least 3 years.

Patients and methods Twelve patients had 14 NETs with a mean 13.4-mm size (10-20) and 17 patients had a cystic tumor (16 IPMN, 1 MCA) with a 29.1-mm mean size (9-60 were included. They were treated with EUS-guided RFA, evaluated prospectively at 1 year, and followed annually for at least 3 years.

Results The mean duration of follow-up was 42.9 months (36-53). Four patients died during follow-up (17-42 months) from unrelated diseases.

At 1-year follow-up, and $85.7 \%$ complete disappearance was seen in 12 patients with 14 NETs. At the end of followup (45.6 months), complete disappearance of tumors was seen in $85.7 \%$ of cases. One case of late liver metastasis occurred in a patient with initial failure of EUS-RFA. At 1-year follow-up, a significant response was seen in $70.5 \%$ of 15 patients with PCNs. At the end of the follow-up, there was a significant response in $66.6 \%$ with no mural nodules. Two cases of distant pancreatic adenocarcinoma unrelated to IPMN occurred.

Conclusions EUS-RFA results for pancreatic NETs or PCNs appear to be stable during 42 months of follow-up.

\section{Introduction}

Endoscopic ultrasound-guided radiofrequency ablation (EUSRFA) has recently emerged as a new technique for pancreatic tumor ablation, mainly for neuroendocrine tumors (NETs), pancreatic cystic neoplasms (PCNs), or pancreatic adenocarcinoma. NETs are probably the indication of choice for EUS-guided
RFA. NETs are mostly non-functional and do not induce a secretory disorder [1]. Once their nature is determined with diagnostic tests like EUS-FNA, incidental nonfunctional NETs currently lead to difficult management when their largest diameter is $<2 \mathrm{~cm}[2-4]$. In patients with tumors ranging from 1 to $2 \mathrm{~cm}$, EUS-guided treatment may be an alternative to surgery [5-7]. PCNs are common pancreatic lesions that are mainly discov- 
ered fortuitously [8-11]. Most PCNs, including intraductal papillary mucinous neoplasms (IPMNs) and mucinous cystadenoma (MCAs), have a benign pattern, and few of them transform to become malignant. Branch duct IPMNs develop malignancy in about $5 \%$ to $10 \%$ of cases, requiring imaging follow-up [2]. PCNs presenting at imaging with worrisome features or highrisk stigmata (WF/HRS) (presence of mural nodules $>5 \mathrm{~mm}$ and cyst size $>3 \mathrm{~cm}$ ) are considered high-risk factors for malignancy [9-11]. An interesting alternative to surgery could be the destruction using EUS-RFA [12-15].

About five series have been published, including NETs or PCNs, most of them being retrospective or including only a few patients [16-20]. In addition, the follow-up of the patients in these series is $<13$ months [16-20]. The short-term efficacy in these series ranges between $71.4 \%$ and $100 \%$ for NETs and around $60 \%$ for PCN but the long-term outcome and risk of recurrence have been unknown until now [16-20]. Although these results regarding short-term efficacy are interesting, surgery remains the treatment of choice in the absence of longterm follow-up and randomized series. However, regarding the results of surgical resection of benign tumors like NETs, the mortality rates range from $3 \%$ to $14 \%$ and morbidity rates from $15 \%$ to $30 \%$ compared to no mortality and morbidity ranging from $3 \%$ to $10 \%$ with EUS-RFA [21,22].

Studies assessing the results of EUS- RFA were conducted between 2015 and 2019 [16-20]. Of them, three were prospective studies with follow-up ranging from 6 months to 1 year $[16,18,19]$. Our team conducted a multicenter prospective study between February 2015 and February 2017 for which 1-year follow-up was published [18], including patients with NETs or PCNs. Because the last patient included has now had at least 3 years of follow-up, we decided to assess the long-term outcome of these patients with follow-up ranging from 3 to 5 years prospectively.

The primary objective of this series was to assess in patients with at least 3 years follow-up the efficacy of EUS-RFA and the secondary objective was to assess the risk of recurrence and outcomes in patients with complications.

\section{Patients and methods}

\section{Patients and criteria for inclusion}

This prospective, multicenter, non-randomized study started in 2015 and included 30 patients with NETs or PCNs. It received the approval of an independent ethics committee (Comité de protection des personnes Sud Méditerranée I; November 17th 2014; reference number 2014-A01474-43) and received ClinicalTrials.gov ID registration: NCT02330497

The included patients presented with either pancreatic NETs $<2 \mathrm{~cm}$ on computed tomography (CT) scan with histological proof (EUS-FNA result yielded endocrine tumor belonging to the G1 WHO classification) or branch duct IPMN (connecting duct identified on magnetic resonance imaging [MRI] and/or EUS) with WF/HRS including mural nodules $>5 \mathrm{~mm}$ (HRS) or MCA. The patients were non-operable due to severe comorbidity or they refused surgery that was offered as first-line treatment. The patients were 16 males and 14 females with a mean age of 54.4 years (49-84 years), 12 with NETs and 17 with PCNs ( Table 1). One patient who had a final diagnosis of late pancreatic metastasis of renal carcinoma was excluded.

The patients were included over 2 years from February 2015 and were followed up during the 3 years after inclusion. At 1 year, recorded data were analyzed and published [18]. Followup included repeated EUS and CT/MRI every year.

\section{Objectives and endpoints}

The primary objective was the antitumor efficacy after at least 3 years of follow-up. The criteria for efficacy in PCNs were complete necrosis, diameter decrease $>50 \%$, diameter decrease $<50 \%$, and the disappearance of mural nodules assessed by EUS/MRI ( Fig. 1). The diagnosis of disappearance or necrosis in NETs was made at the CT scan according to the lesion's disappearance or the absence of contrast enhancement at the arterial time in a low-density lesion ( $\triangleright$ Fig.2). Contrast-enhanced harmonic (CEH) EUS was not used in all the centers included in the study. Results were classified as complete resolution (disappearance or necrosis), significant response (decrease $>50 \%$ or complete resolution), failure (decrease $<50 \%$ or no effect).

The secondary objectives were to assess the recurrence on the treated site or other sites and the outcome of patients with complications. In the case of recurrence, a second session was proposed to the patient in the absence of malignancy.

- Table 1 Characteristics of lesions treated with EUS-RFA

\begin{tabular}{|c|c|c|c|}
\hline Type of lesion & Location & Diameter (mean, range) & Special findings \\
\hline $\begin{array}{l}\text { NET } \\
12 \text { patients }\end{array}$ & $\begin{array}{l}\text { Head } 3 \\
\text { Body } 6 \\
\text { Tail } 5\end{array}$ & $13.4 \mathrm{~mm}(10-20 \mathrm{~mm})$ & $\begin{array}{l}2 \text { cystic NETs } \\
2 \text { double NETs (1 MEN 1) }\end{array}$ \\
\hline $\begin{array}{l}\text { PCN } \\
17 \text { patients (16 IPMN, } 1 \mathrm{MCA})\end{array}$ & $\begin{array}{l}\text { Head } 10 \\
\text { Body } 4 \\
\text { Tail } 3\end{array}$ & $29.1 \mathrm{~mm}(19-60 \mathrm{~mm})$ & $\begin{array}{l}12 \text { mural nodules }>5 \mathrm{~mm} \\
4 \text { increased thickness wall } \\
5 \text { multiloculated cysts }\end{array}$ \\
\hline
\end{tabular}

NET, neuroendocrine tumor; PCN, pancreatic cystic neoplasm; IPMN, intraductal pancreatic mucinous neoplasm; MCA, mucinous cystadenoma. 

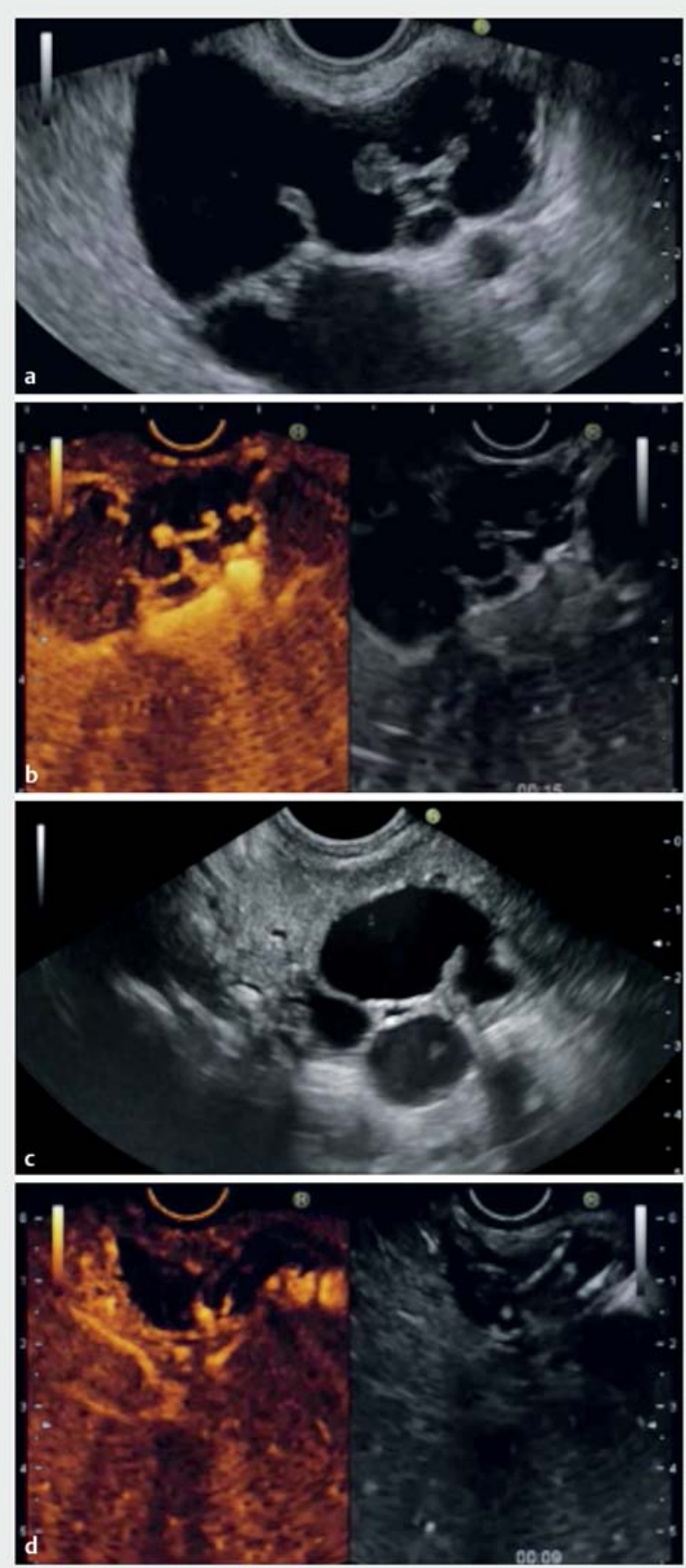

- Fig. 1 EUS-guided RFA for IPMN with mural nodules. a EUS view showing a large IPMN with mural nodules located in the body of the pancreas. $\mathbf{b}$ CEH EUS showing enhancement of the mural nodules. c Follow-up at 42 months after EUS-RFA showing no disappearance of the cystic lesion but complete disappearance of mural nodules. d Follow-up at 42 months with CEH EUS showing no enhancement of mural nodule.

\section{Technique}

All endoscopic examinations were performed with an EUS therapeutic scope (EG-3870UTK, HOYA corporation Pentax life care division, Tokyo, Japan, or GF-UCT180 Olympus, Tokyo, Japan). In the case of NET, the operative needle was used directly. In the case of PCN, fine-needle aspiration (FNA) with a $22 \mathrm{G}$ regular needle was performed first to suck the fluid content until a thin layer of fluid content remains to target the remaining cystic lesion, then the operative needle was used. If the fluid content was too viscous, the $22 \mathrm{G}$ fine needle was exchanged with a 19 G FNA needle. In the case of multiloculated cysts, septations were torn with fanning movement of the needle tip to suck the main part of the fluid content.

EUS-RFA was performed with an $18 \mathrm{G}$ RFA needle (STARmed, Taewoong, Korea) applying a $50 \mathrm{~W}$ current with Continuance Mode setting until reaching 100 Ohms impedance (white bubble appearance) and not exceeding 500 Ohms. RFA was stopped either when the operator saw white bubbles on the EUS screen alongside the needle and outside the targeted lesions or when the impedance exceeded 100 Ohms. With the STARmed needle, the mean duration of radiofrequency exposure was related to both impedance surveillance and white bubble diffusion, usually ranging between 20 and 45 seconds.

\section{Prophylaxis for complications}

Prophylaxis was administered for acute pancreatitis, infection, and perforation following complications in the first two patients [18]. Rectal diclofenac was given as recommended before endoscopic retrograde cholangiopancreatography to prevent post-endoscopic pancreatitis. Antibiotic prophylaxis $(2 \mathrm{~g}$ of amoxicillin and clavulanic acid intravenously) was given to avoid infection. Sucking the main part of the fluid content in the PCN procedure was performed previously before RFA to avoid excessive radiofrequency current into the liquid component.

\section{Results}

\section{Patients and follow-up}

At inclusion, there were 12 patients with 14 NETs and 17 patients with PCNs (16 IPMN and 1 MCA).

NET patients were seven males (58\%) and five females with a mean age of 59.9 years (45-77). All were well-differentiated and non-functional NETs belonging to OMS grade 1 classification and associated, in one case, with multiple endocrine neoplasia (MEN) type 1 . The mean size of the NETs was $13.1 \mathrm{~mm}$ $(10-20 \mathrm{~mm})$. Their location was three in the head, six in the body, and five in the tail. PCN patients had branch duct IPMNs in 16 cases and one MCA. They were seven males and $10 \mathrm{fe}-$ males $(44 \% / 56 \%)$ with a mean age of 65.7 years $(65-83)$. The mean diameter size was $28 \mathrm{~mm}(9-60 \mathrm{~mm})$. They were located on the head in 10 cases, the body in four cases, and the tail in three cases. Among 16 cases with branch duct IPMNs, 12 patients had mural nodules (mean size $5.7 \mathrm{~mm}$ [5-10 mm]) and with increased thickness of the cyst wall in 4 cases (mean size $3.3 \mathrm{~mm}[2-7 \mathrm{~mm}])$. 



- Fig. 2 EUS-guided RFA with CEH control for NET located to the body of the pancreas. a EUS view of NET located to the body of the pancreas, $11 \mathrm{~mm}$ size, homogenous. b EUS view of NET with fine doppler showing peripheral arterial vascularization. c CEH EUS view of NET showing early hyperenhancement. d immediate CEH EUS view of NET after EUS-RFA showing no more enhancement of the treated NET.

- Table 2 NET follow-up

\begin{tabular}{|l|l|l|l|}
\hline Inclusion & 1-year follow-up & $\mathbf{> 3}$-year follow-up & Final results \\
\hline $\begin{array}{l}14 \text { NETS } \\
12 \text { patients }\end{array}$ & $\begin{array}{l}\text { 11 successes } \\
1 \text { late recurrence }\end{array}$ & Success $85.7 \%$ \\
\hline & 2 disappearance & $\begin{array}{l}1 \text { late success } \\
1 \text { metastatic evolution }\end{array}$ & Failure $14.3 \%$ \\
\hline
\end{tabular}

NET, neuroendocrine tumor.

Patients were followed up at least 3 years after inclusion, the last one being included in February 2017. The mean age at the end of the follow-up was 62.5 years (52-87 years). Mean duration of follow-up was 42.9 months (36-53). Four patients died during the follow-up. There were two males and two females with ages ranging between 52 years to 77 years. They died respectively at $42,42,17$, and 26 months from different causes than from the NETs or IPMNs: one suicide in a patient with MEN1 and NET; one of a hemorrhagic stroke, one of hepatocelIular carcinoma; and one developed a distant pancreatic cancer in the tail of the pancreas after being treated with EUS- RFA for IPMN located in the head of the pancreas (without cystic lesions in the tail of the pancreas).

\section{Long-term efficacy}

Results in NETs

All of the patients were followed up for at least 3 years with a mean of 45.6 months, despite one patient who died at 42 months follow-up from suicide, with complete disappearance of the two NETs treated ( $\triangleright$ Table 2 ).

At 1-year follow-up, 12 NETs had completely disappeared or appeared necrotic (85.7\%) ( $\mathbf{F i g . 2}$ ). Two were considered a failure.

At the end of the follow-up (45.6 months), 12 NETs had completely disappeared (85.7\%). One of them had a small hyperechoic scar after 6 months of follow-up without contrast enhancement on CT scan and EUS with negative FNA. The scar remained stable from 6 to 41 months follow-up. One of them was initially considered as a failure at 1 year because the cystic lesion, which was located at the tail of the pancreas, still measured $20 \mathrm{~mm}$. No contrast enhancement was seen on EUS. At 
the end of follow-up (53 months), the lesion disappeared entirely on CT scan and EUS.

\section{Failure and second EUS RFA session}

There were two failures, of which one was a recurrence after disappearance at 1 year; another one was a failure at 1 -year follow-up. One was a NET occurring in a 73-year-old male patient. The lesion was located in the body of the pancreas, and measured $18 \mathrm{~mm}$ at inclusion. At 6 months, the lesion was $13 \mathrm{~mm}$, and at 1 year, the lesion could not be found anymore, considered as a success. At the end of the follow-up (42 months), the lesion was again measured at $16 \mathrm{~mm}$ on EUS with no contrast enhancement at contrast-enhance harmonic EUS. The FNA showed well-differentiated neuroendocrine cells, G1 of the WHO classification. A second session was scheduled for the patient, but he refused.

The second one occurred in a 65-year-old female patient. The lesion was located in the body of the pancreas and measured $19 \mathrm{~mm}$ at inclusion with a G1 WHO classification. Treatment was considered a failure at 1 year with the lesion measuring $12 \mathrm{~mm}$ at 6 months and $16 \mathrm{~mm}$ at 1 year. The patient received a second session of EUS-RFA at 23 months. A CT scan at 2years follow-up showed no liver metastasis and the persisting lesion measured $13 \mathrm{~mm}$ in the body of the pancreas. On the CT scan, at 3-year follow-up, asymptomatic liver metastases were shown on a positron emission tomography scan that identified intense uptake on liver metastases and pancreatic tail. The patient is doing well and receives somatostatin therapy. The second report of the pathological examination of the first FNA was corrected from G1 to G2 of the WHO classification.

\section{Results in pancreatic cystic neoplasms (PCNs)}

Patients treated for PCNs were followed for a mean of 42.6 months and two were excluded because they died at 17 and 26 months, respectively. One died from hepatocellular carcinoma related to liver cirrhosis associated with alcoholism. He had a 76-mm branch duct IPMN with mural nodule and high-grade dysplasia at inclusion. At the end of follow-up, the IPMN measured $60 \mathrm{~mm}$ with no mural nodule. The second patient died at 26 months from a distant pancreatic adenocarcinoma not related to the side branch IPMN that was treated.

At 1 year with 17 patients, 11 had complete disappearance (64.7\%), and one diameter decreased by more than $50 \%$. The response rate was $70.5 \%$. Four cases presented with a diameter decrease $<50 \%$ and one with no changes. Mural nodes all disappeared at EUS follow-up in 12 of 12 cases (100\%).

At the end of 42.6-month follow-up on 15 patients, six (40\%) had complete disappearance of the lesion with an initial median size of $18 \mathrm{~mm}$ (range $11-37 \mathrm{~mm}$ ). Four patients $(26.6 \%)$ had a decrease $>50 \%$ with an initial median size of $12 \mathrm{~mm}$ (range 9$32 \mathrm{~mm}$ ) changing to $4-\mathrm{mm}$ median size $(4-16 \mathrm{~mm})$. Three of them were small recurrences with size ranging between 4 and $6 \mathrm{~mm}$.

In five patients, treatment was a failure or lesions decreased $<50 \%$ with an initial median size of $35 \mathrm{~mm}(25-76 \mathrm{~mm})$ changing to $30-\mathrm{mm}$ median size $(25-60 \mathrm{~mm})$. Three had no growth increase, two a slight size decrease.
In all cases, the 10 patients with mural nodules at inclusion had no more mural nodules (3, 4, 3 had complete disappearance, $>50 \%$ decrease, and $<50 \%$ decrease, resspectively) (> Fig. 1).

\section{Second EUS-RFA session}

Two patients underwent a second EUS-RFA session. One had a decrease in tumor size $>50 \%$ (32 $\mathrm{mm}$ to $12 \mathrm{~mm}$ ) and complete disappearance of the mural nodule. One had no change in size $(25 \mathrm{~mm})$ of a PCN located in the head and she underwent a second session at 19 months. A severe complication occurred (biliary leakage endoscopically managed) after seven RFA shots had been given with no effect on the tumor size.

\section{Distant pancreatic adenocarcinoma}

One patient died from a hemorrhagic stroke during follow-up at 42 months (>3 years) and, therefore, was not excluded. That person had an IPMN located in the head of the pancreas that measured $40 \mathrm{~mm}$. At 1 year, the IPMN remained stable in but without a mural nodule. At the end of 42-months follow-up, there was no cystic lesion located in the head of the pancreas and complete disappearance of the previous one. Nevertheless, a pancreatic adenocarcinoma appeared in the tail of the pancreas without a previous cystic lesion. The patient was scheduled to have a left pancreatectomy, but he died from a hemorrhagic stroke.

The second patient died at 26 months from distant pancreatic carcinoma located in the tail of the pancreas with liver metastases. He was included for management of branch duct IPMN located in the head of the pancreas that measured 46 $\mathrm{mm}$ with a $6-\mathrm{mm}$ mural nodule. At that time, there was no cystic lesion located in the tail of the pancreas. At 1 year and 26 months, the cystic lesion located in the head of the pancreas measured $10 \mathrm{~mm}$ with no mural nodule.

\section{Overall complications}

Three patients had immediate complications following EUSRFA: one had pancreatitis, which was conservatively managed; one had a perforation that was surgically managed with suturing and a favorable outcome with complete disappearance of the IPMN; one had pancreatic duct stenosis that was endoscopically managed no late symptoms. All had full recovery without sequelae and total disappearance of the initial lesions (2 NETs in the first case, IPMN with high-grade dysplasia in the second case, NET in the third case).

One patient had a biliary leakage after a second RFA session at 19 months. She had biliary leakage treated with EUS-guided cyst-gastrostomy and biliary stenting. One year later, all the stents were removed with no sequelae but with no change in the size of the IPMN.

The overall complication rate was four of 29 patients (13.79\%). When the protocol changes after the two first complicated patients (nonsteroidal anti-inflammatory drug, antibiotic prophylaxis, sucking the fluid content) were considered, the rate was reduced to $7.4 \%$.

No delayed adverse events related to the initial RFA session occurred during the follow-up. 


\section{Discussion}

An increasing number of studies evaluating EUS-RFA for treating premalignant pancreatic tumors such as NETs and PCNs are available. Most of them have included a small number of patients and some are retrospective [16-20]. Five series about EUS-RFA of NETs have been published [16-20], three of them prospective, including one to 12 patients [16-20,23, 24]. Three studies about EUS-RFA of PCNs have been published, two prospective, including 6 to 17 patients $[16,18,25]$. In addition, the follow-up was limited to 1 year (10-13 months). If the efficacy for NETs ranged between $71.4 \%$ and $100 \%$ and for PCNs was between $48.4 \%$ and $65 \%$, the long-term results are still unknown and the clinicians cannot rule out the risk of recurrence or unfavorable outcomes such as early malignancy [18, 23-25].

We decided to extend the follow-up of the prospective multicenter study that started in February 2015 and ended in February 2017, with 1-year follow-up. All the patients were checked annually at least 3 years after inclusion, with a mean follow-up of 42.9 months (36-53). Four patients died during follow-up due to unrelated diseases ( 1 suicide, 1 hepatocellular carcinoma, 1 hemorrhagic stroke, and 1 distant pancreatic cancer). The latter was a distinct pancreatic adenocarcinoma located in the tail, whereas the IPMN was in the head of the pancreas, and probably developed on ductal PanIN 3 lesions. In all of the patients, controlled was achieved with EUS at the end of follow-up, as shown in MRI in the case of PCN or CT scan/MRI in the case of NET.

Regarding the RFA results for NETs, the long-term results of EUS- RFA in 14 lesions occurring in 12 patients appeared to be stable, with $85.7 \%$ at 1 year and the end of follow-up ( $\triangleright$ Table 2 ). These apparently stable results included one late response and one late recurrence. The late response was a case considered as a failure at 1 year despite negative CEH EUS; the tumor disappeared completely at 53-month follow-up. We postulate that it could be a delayed success related to the activated immune response associated with RFA [18-26]. Conversely, an 18-mm NET located in the body of the pancreas seemed to disappear at 1 year after the size decreased to $13 \mathrm{~mm}$ at 6 months. It reoccurred at 42-month follow-up at a size of $16 \mathrm{~mm}$. No contrast enhancement could be seen on CEH EUS, but EUS-FNA showed well-differentiated endocrine cells belonging to the G1 WHO classification. We have no clear explanation for this late and benign recurrence.

Although the protocol of this study included only one RFA shot with $50 \mathrm{~W}$ not exceeding 100 Ohms, we now apply one to three shots, depending on the results of immediate CEH EUS ( $\vee$ Fig.2). After considering the complete diffusion of the white bubbles into the volume of the tumor, the tumor was assessed by CEH EUS. In the case of persisting contrast enhancement, we added a targeted RFA shot. The same protocol also has been supported by other authors [23, 24, 27]. A recent series demonstrated in 19 patients with solid abdominal tumor the usefulness of targeting EUS- RFA with CEH-EUS findings [27]. Nevertheless, in the protocol for the study we began in 2015, CEH EUS was not required in all the centers included because it was not available in all the institutions.
The risk for developing metastatic lymph nodes or liver metastasis during follow-up of NETs is probably the main uncertainty of EUS-RFA management. Unfortunately, in our series, one case showed an unfavorable outcome with metastasis in a 65-year-old female. The lesion was classified as G1 of the WHO classification, located in the body of the pancreas, and measured $19 \mathrm{~mm}$. Her treatment was considered a failure at 1 year. She received a second session of EUS-RFA at 23 months and the size of the lesion decreased to $13 \mathrm{~mm}$. She developed asymptomatic liver metastases at 36 months with a PET scan identifying an intense uptake on liver metastases and in the pancreatic tail. Because we did not understand the metastatic outcome, we asked for a second opinion on the pathological examination of the first FNA. It was corrected from G1 to G2 of the WHO classification. It has been shown in the literature that the accuracy of FNA for adequate WHO classification ranges from $74 \%$ to $87.5 \%$ [28]. This could explain the discrepancy between the two pathological reports, which underscores the need for long-term follow-up. In case of failure at 1 year, surgery should be recommended despite the fact that delayed healing can occur after a 1-year follow-up. The risk of developing further malignancy during the follow-up of NETs $<2 \mathrm{~cm}$ is estimated to be up to $27.3 \%$ for lymph nodes and $9 \%$ for metastases [29]. The European NET guidelines and the revised National Comprehensive Cancer Network guidelines suggest surveillance for lesions $<2 \mathrm{~cm}$ (and no more surgery) [30-32]. Pancreatic surgery for NETs is associated with a mortality rate ranging between $3 \%$ to $6 \%$ and morbidity rate ranging from $14 \%$ to $58 \%$, mainly due to pancreatic fistula [21]. The 5-year overall survival and diseasespecific survival rates are $85 \%$ and $93 \%$, respectively [21]. A randomized series comparing surgical resection and EUS-RFA should be conducted. Looking at the metastatic outcome in this patient, we recommend referring the patient to surgery in case of failure of EUS-RFA at 1 year.

Regarding the PCN results, the disappearance and size of the tumor showed relative stability, whereas all the mural nodules disappeared ( $\triangleright$ Table 3 ). A significant response (disappearance or size decreasing $>50 \%$ ) was shown in $70.5 \%$ at 1 year versus $66.6 \%$ at the end of the follow-up ( $\triangleright$ Table 3 ). Three patients presented with recurrence of a small cyst ranging between 4 and $6 \mathrm{~mm}$. RFA failures remained stable, occurring in one-third of patients. The initial median size in patients with failure was greater than those with disappearance or size decrease $>50 \%$ $(\triangleright$ Table 3 ). Even in patients with failure, three had a mural nodule, which disappeared entirely. As for EUS-RFA, the initial protocol called for a one-shot treatment within the cystic lesions. After the end of this study, we applied two to five shots for filling the lumen of the cyst with white bubbles. We never exceeded five shots because we had a biliary leakage in the patient with IPMNs located in the head of the pancreas who had undergone seven shots in a second RFA session at 19-month followup. The biliary leakage was resolved with biliary stenting for 1 year with no stenosis.

Two cases of distant pancreatic adenocarcinoma occurred in the tail of the pancreas, whereas both of the IPMN lesions were located in the head of the pancreas. One patient from liver metastasis and pancreatic adenocarcinoma in the tail of the pan- 


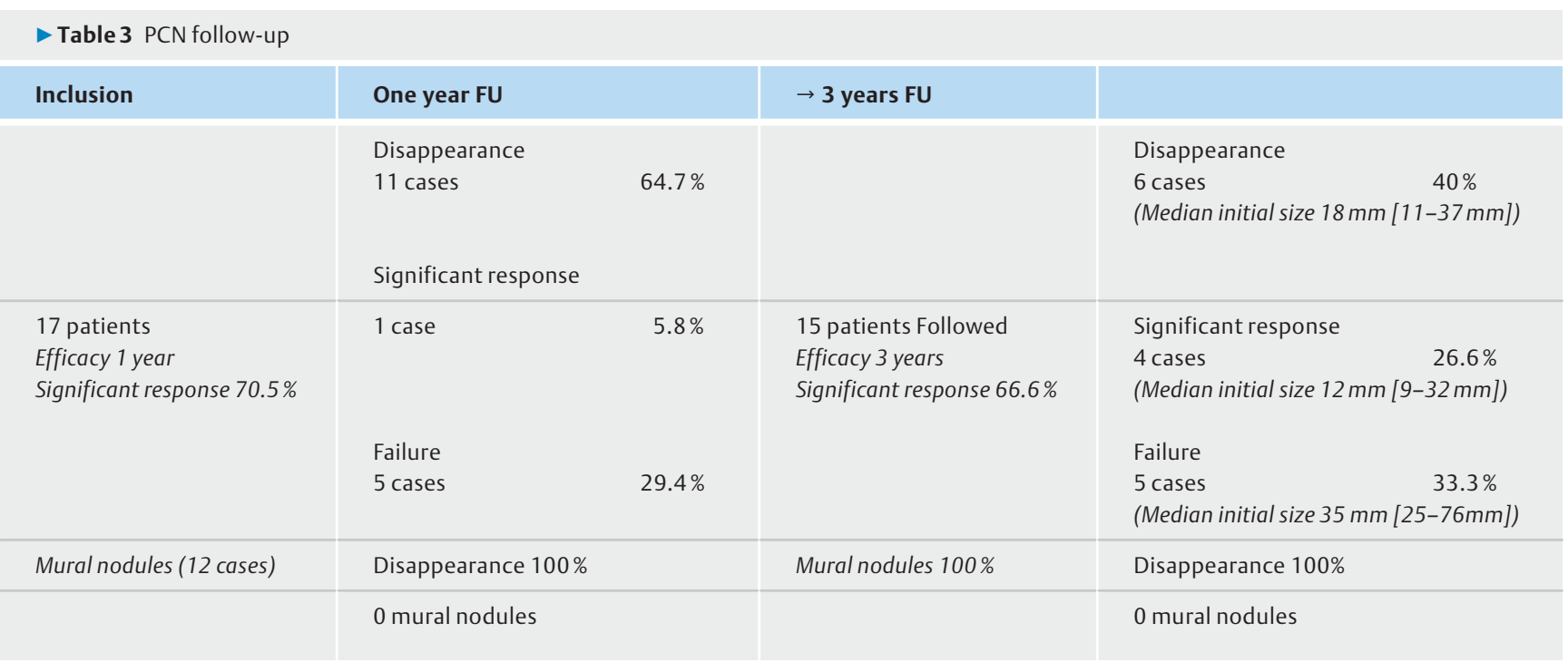

creas at 26 months, whereas the second one died at 42 months from a stroke before undergoing left pancreatectomy. The association of IPMNs with pancreatic adenocarcinoma arising from PanIN3 lesions distant from the cystic lesion has been described in the literature [33-36]. The 5-year rate of development of distinct adenocarcinoma in patients with IPMNs has been calculated to $6.9 \%$, with an incidence per year of $1.1 \%$ [33]. In a surgical series of 40 patients with IPMNs, pancreatic intraepithelial neoplasia was found in $78 \%, 11 \%$ being PanIN-3 lesions [35]. EUS seems to be able to detect $69 \%$ of PanIN lesions, $57 \%$ with PanIN-3 lesions [35]. In another series assessing the role of EUS, the 3- and 5-year rates of IPMN-concomitant pancreatic ductal adenocarcinoma were $4 \%$ and $8.8 \%$, respectively [36]. The EUS strategy should include not only the therapeutic approach with RFA but also sustained surveillance, not only of the treated cystic lesion but also of distinct and distant lesions, such as in these two patients.

\section{Conclusions}

In conclusion, EUS-RFA management of pancreatic NETs or PCNs is associated with significant positive long-term outcomes. The complete disappearance of NETs occurred in $85.7 \%$ of cases. Treatment was a failure in only one patient, who did not undergo surgery because of metastasis. For PCNs, a significant response occurred in $66.6 \%$ of patients, with complete disappearance of all mural nodules. However, two patients developed distinct and distant pancreatic adenocarcinoma, which underscores the need for accurate and sustained EUS follow-up.

\section{Competing interests}

Dr. Barthet received a research grant from Boston scientific (endoscopic gastrojejunal anastomosis). Dr. Napoleon is a consultant for Boston Scientific.
References

[1] Lee DW, Kim MK, Kim HG. Diagnosis of Pancreatic Neuroendocrine Tumors. Clin Endosc 2017; 50: 537-545

[2] Sachs T, Pratt WB, Callery MP et al. The incidental asymptomatic pancreatic lesion: nuisance or threat? J Gastrointest Surg Off J Soc Surg Aliment Tract 2009; 13: 405-415

[3] Strosberg JR, Cheema A, Weber J et al. Prognostic validity of a novel American Joint Committee on Cancer Staging Classification for pancreatic neuroendocrine tumors. J Clin Oncol Off J Am Soc Clin Oncol 2011; 29: 3044-3049

[4] Metz DC, Jensen RT. Gastrointestinal neuroendocrine tumors: pancreatic endocrine tumors. Gastroenterology 2008; 135: 1469-1492

[5] Finks JF, Osborne NH, Birkmeyer JD. Trends in hospital volume and operative mortality for high-risk surgery. N Engl J Med 2011; 364: 2128-2137

[6] Lakhtakia S. Therapy of pancreatic neuroendocrine tumors: fine needle intervention including ethanol and radiofrequency ablation. Clin Endosc 2017; 50: 546-551

[7] Chennat J. Current status of endoscopic ultrasound guided ablation techniques. Gastroenterology 2011; 140: 1403-1409

[8] Farrell J]. Prevalence, diagnosis and management of pancreatic cystic neoplasms: current status and future directions. Gut Liver 2015; 9 : 571-589

[9] Tanaka M, Fernández-Del Castillo C, Kamisawa T et al. Revisions of international consensus Fukuoka guidelines for the management of IPMN of the pancreas. Pancreatol Off I Int Assoc Pancreatol IAP AI 2017; 17: 738-753

[10] Brugge WR, Lewandrowski K, Lee-Lewandrowski E et al. Diagnosis of pancreatic cystic neoplasms: a report of the cooperative pancreatic cyst study. Gastroenterology 2004; 126: 1330-1336

[11] Okabayashi T, Kobayashi M, Nishimori I et al. Clinicopathological features and medical management of intraductal papillary mucinous neoplasms. J Gastroenterol Hepatol 2006; 21: 462-467

[12] Lakhtakia S, Seo D-W. Endoscopic ultrasonography-guided tumor ablation. Dig Endosc Off J Jpn Gastroenterol Endosc Soc 2017; 29: 486-494 
[13] Zacharoulis D, Lazoura O, Rountas $C$ et al. Experimental animal study of a novel radiofrequency endovascular occlusion device. Am J Surg 2011; 202: 103-109

[14] Sethi A, Ellrichmann M, Dhar S et al. Endoscopic ultrasound-guided lymph node ablation with a novel radiofrequency ablation probe: feasibility study in an acute porcine model. Endoscopy 2014; 46: 411415

[15] Kim HJ, Seo D-W, Hassanuddin A et al. EUS-guided radiofrequency ablation of the porcine pancreas. Gastrointest Endosc 2012; 76: 1039-1043

[16] Pai M, Habib N, Senturk H et al. Endoscopic ultrasound guided radiofrequency ablation, for pancreatic cystic neoplasms and neuroendocrine tumors. World J Gastrointest Surg 2015; 7: 52-59

[17] Lakhtakia S, Ramchandani M, Galasso D et al. EUS-guided radiofrequency ablation for management of pancreatic insulinoma by using a novel needle electrode (with videos). Gastrointest Endosc 2016; 83: 234-239

[18] Barthet M, Giovannini M, Lesavre N et al. Endoscopic ultrasound guided radiofrequency ablation for pancreatic neuroendocrine tumors and pancreatic cystic neoplasms: a prospective multicenter study. Endoscopy 2019; 51: 836-842

[19] Choi JHS, Song TJ, Park DH et al. Endoscopic ultrasound-guided radiofrequency ablation for management of benign solid pancreatic tumors. Endoscopy 2018; 50: 1099-1104

[20] Oleinikov KD, Epshtein J, Benson A et al. Endoscopic ultrasoundguided radiofrequency ablation: a new therapeutic approach for pancreatic neuroendocrine tumors. J Clin Endoc Metab 2019; 104: 2637-47

[21] Jilesen APJ, Van Eijck CHJ, in't Hof KH et al. Postoperative complications, In-Hospital mortality and 5-year survival after surgical resection for patients with a pancreatic neuroendocrine tumor: a systematic review. World J Surg 2016; 40: 729-748

[22] Feng Q, Li C, Zhang S et al. Recurrence and survival after surgery for pancreatic cancer with or without pancreatitis. World J Gastroenterol 2019; 25: 6006-6015

[23] Rimbas M, Horumba M, Rizzatti G et al. Interventional endoscopic ultrasound for pancreatic neuroendocrine neoplasms. Dig Endosc 2020; 32: 1031-1041

[24] Larghi A, Rizzatti G, Rimbas M et al. Endoscopic ultrasound-guided radiofrequency ablation as an alternative to surgery for pancreatic neuroendocrine neoplasms: Who should we treat? Endosc Ultrasound 2019; 8: 220-226

[25] Canakis A, Law R, Baron T. An updated review on ablative treatment of pancreatic cystic lesions. Gastrointest Endosc 2020; 91: 520-526

[26] Slovak R, Ludwig JM, Gettinger SN et al. Immuno-thermal ablations boosting the anticancer immune response. I Immunother Cancer 2017; 5: 78

[27] Choi JH, Seo DW, Song TJ et al. Utility of contrast-enhanced harmonic endoscopic ultrasound for the guidance and monitoring of endoscopic radiofrequency ablation. Gut Liver 2020; 14: 826-832

[28] Sugimoto M, Takagi T, Hikichi T et al. Efficacy of endoscopic ultrasonography-guided fine needle aspiration for pancreatic neuroendocrine tumor grading. World J Gastroenterol 2015; 21: 8818-8124

[29] Lombardi M, De Lio N, Funel M et al. Prognostic factors for pancreatic neuroendocrine neoplasms (pNET) and the risk of small non-functional pNET. J Endocrinol Invest 2015; 38: 605-613

[30] Falconi M, Eriksson B, Kaltsas $G$ et al. ENETS consensus guidelines update for the management of patients with pancreatic functional neuroendocrine tumors and pancreatic non-functional neuroendocrine tumors. Neuroendocrinology 2016; 103: 153-171

[31] Teo RYA, Teo TZ, Tai DWM et al. Systematic review of current prognostication systems for pancreatic neuroendocrine neoplasms. Surgery 2019; 165: 672-685

[32] Choi JH, Choi YH, Kang J et al. Natural history of small pancreatic lesions suspected to be non -functioning pancreatic neuroendocrine tumors. Pancreas 2018; 47: 1358-1364

[33] Uehara H, Nakazaizumi A, Ishikawa O et al. Development of ductal adenocarcinoma of the pancreas during follow-up of branch duct intraductal papillary mucinous neoplasms of the pancreas. Gut 2008; 57: 1561-1566

[34] Sahora K, Crippa S, Zamboni G et al. Intraductal papillary mucinous neoplasms of the pancreas with concurrent pancreatic and periampullary neoplasms. Eur J Surg Oncol 2016; 42: 197204

[35] Maire F, Couvelard A, Palazzo L et al. Pancreatic intraepithelial neoplasia in patients with intraductal papillary mucinous neoplasms: the interest of endoscopic ultrasonography. Pancreas 2013; 42: 12621266

[36] Kamata K, Kitano M, Kudo M et al. Value of EUS in early detection of pancreatic ductal adenocarcinomas in patients with intraductal papillary mucinous neoplasms. Endoscopy 2014; 46: 22-29 